\title{
RADIAL VELOCITY AND MAGNETIC VARIATIONS OF $\alpha$ DRACONIS
}

Holger Lehmann, Thüringer Landessternwarte, Karl-Schwarzschild-Observatorium, 0-6901 Tautenburg, Germany

Gerhard Scholz, Astrophysikalisches Institut Potsdam, Telegrafenberg, 0-1561 Potsdam, Germany

ABSTRACT Based on photographic zeeman spectra a magnetic field has been detected for the giant star $\alpha$ Dra. One of the two periods derived for the magnetic changes can be interpreted as the half rotational period corresponding to a slight variation of the radial velocity of 3.57 days. The application of an equatorially symetric rotator model with a magnetic field dominated by the quadrupole moment leads to a good agreement with the observed magnetic variation. The orbital elements of $\alpha$ Dra were newly derived.

\section{INTRODUCTION}

The star $\alpha$ Draconis (HD 123299) is a well-known single-lined spectroscopic binary with a period of 51.4 days. Its spectral type is AO III (Hoffleit 1982), its orbital elements were derived last by Elst a. Nelles (1983). The projected equatorial velocity was determined by slettebak et al. (1975) to $15 \mathrm{~km} / \mathrm{s}$ and by Adelman et al. (1987) to $20 \mathrm{~km} / \mathrm{s}$. According to Adelman the giant star is metal-weak and lies in the $\mathrm{H}-\mathrm{R}$ diagram intermediately between the mercury-manganese and the metalliclined stars. Until now no hints to a magnetic field of $\alpha$ Dra were given in literature.

\section{SPECTRAL ANALYSIS}

Photographic Zeeman (30) and normal (38) spectrograms with a reciprocal linear dispersion of $7.9 \AA$ have been obtained at the Coude spectrograph of the $2 \mathrm{~m}$ telescope in Tautenburg over a period of 13 years. Based mainly on the $\mathrm{MgII}(\lambda=4481 \mathrm{~A})$ line the spectra were investigated in relation to the radial velocity, the line width representing the vsini and the effective magnetic field. 
Radial velocity

The values of the radial velocity show a dominant variation due to the binary motion. Orbital elements were derived by a common least square fit followed by differential corrections varying the orbital period too (Fig. 1a). Except for the ex-

TABLE I The orbital elements of $\alpha$ Dra

\section{Elst and Nelles (1983) this paper}

\begin{tabular}{lclcl}
$P(d)$ & 51.4167 & \multicolumn{2}{c}{$51.4163 \pm 0.0008$} \\
$v_{0}(\mathrm{~km} / 8)$ & -14.0 & \pm 0.2 & -15.0 & \pm 0.3 \\
$R(\mathrm{~km} / \mathrm{s})$ & 49.7 & \pm 0.3 & 48.0 & \pm 0.4 \\
$\mathrm{e}$ & $0.400 \pm 0.006$ & 0.434 & \pm 0.005 \\
$\omega\left({ }^{\circ}\right)$ & 23.2 & \pm 0.8 & 20.9 & \pm 1.8 \\
$T_{0}$ & 2445117.37 & \pm 0.08 & 2442392.6 & \pm 0.1 \\
$\sigma_{(\mathrm{m} / \mathrm{s})}$ & 2.3 & & 1.74 & \\
$\sigma_{*}(\mathrm{~km} / \mathrm{s})$ & & & 1.29 &
\end{tabular}

$(\mathrm{km} / \mathrm{s})$

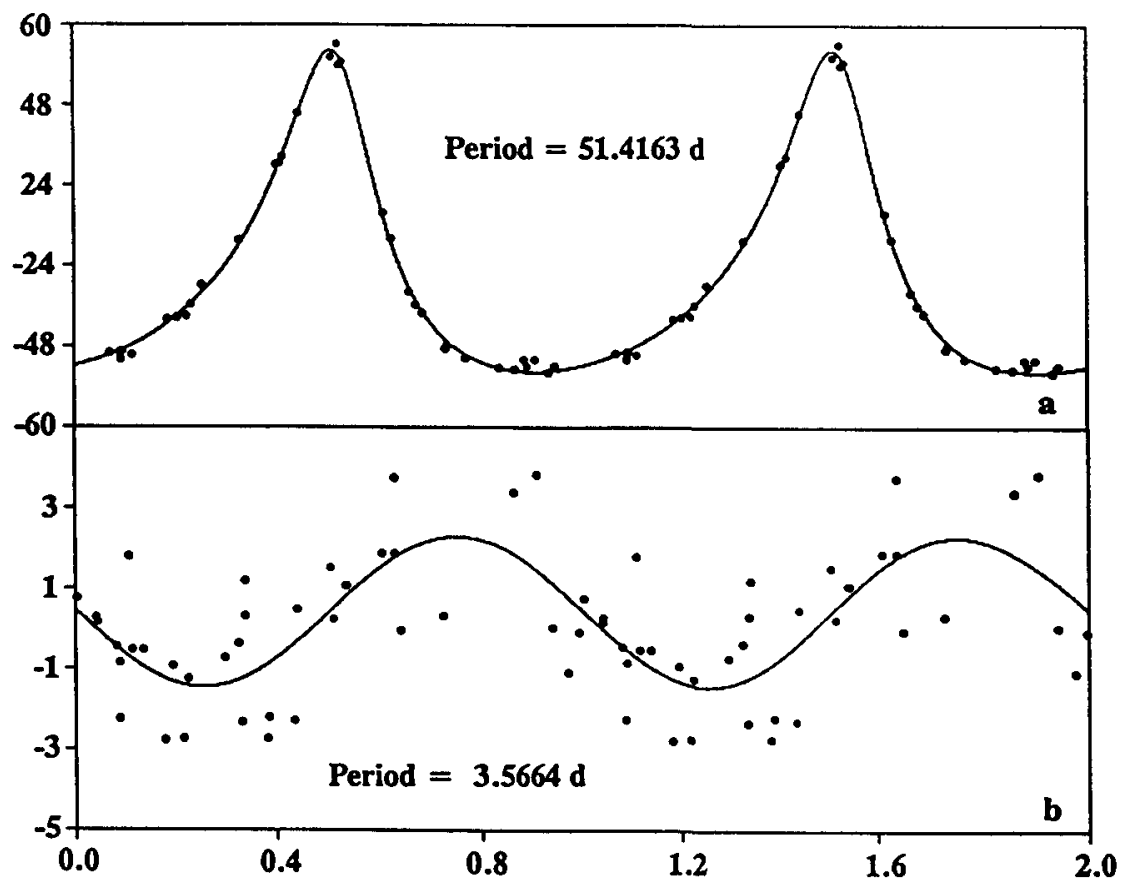

Fig. 1. Radial velocity versus phase. a) mean curve derived from the orbital elements, b) residuals. 
centricity, results are in good agreement with those derived by Elst and Nelles in 1983 (Table I). An analysis of the residuals of the radial velocity after subtracting the mean curve resulting from the orbital elements give hints to only one period at $3.5664 \mathrm{~d}$ (Fig. 1b). In Table I $\sigma$ is the mean deviation of the RV residuals and $\sigma_{\star}$ is the mean deviation after correcting the residuals for the $3.5664 \mathrm{~d}$ period.

\section{vsini}

The profile of the line $\operatorname{MgII}(\lambda=4481 \AA$ ) was analyzed on spectrograms corresponding to the minimum and maximum phase of the measured effective magnetic field strength. The vsini values were derived by means of the Fourier transformation of the intensity profiles followed by a Bessel transformation of the Fourier coefficients (Deeming 1977). The resulting mean value of $27 \mathrm{~km} / \mathrm{s}$ is higher than those of $15 \mathrm{~km} / \mathrm{s}$ resp. $20 \mathrm{~km} / \mathrm{s}$ measured by sletteback et al.(1978) and Adelman et al. (1987, 1989). Although our absolute value of vsini is somewhat uncertain because of the low $S / N$ of the photographic plates there should be no dependence of vsini on time or magnetic field strength exceeding $2 \mathrm{~km} / \mathrm{s}$.

\section{Effective magnetic field}

Only the spectral lines with the best $S / N-\operatorname{MgII}(\lambda=4481 \AA)$ and CaII(R) - have been used for deriving the magnetic field. obviously the star shows throughout a negative effective field strength up to -4000 Gauss, the minimum field strength is about 0 Gauss. The search for periods assuming a sinusoidal variation give limits at two values: 55.071 days and 1.8076 days (Fig. 2a, 2b).

For the longer period some difficulties arise in respect to an explanation by a rotator model. A rotational period of 55 days and vsini of about $20 \mathrm{~km} / \mathrm{s}$ is in contradiction to the stellar parameters which characterize $\alpha$ Dra as a giant. Even though a relation of binary motion and magnetic field is indicated the delay of the magnetic period compared to the orbital period is hardly to explain. Therefore, we interprete the shorter period of $1.8076 \mathrm{~d}$ as an indicator for the rotation of $\alpha$ Dra.

\section{DISCUSSION}

Regarding the small scale variation of the radial velocity of about $3.6 \mathrm{~d}$ as the rotational period of $\alpha$ Dra the short magnetic period of $1.8076 \mathrm{~d}$ can be intexpreted as a double wave produced by a magnetic quadrupole during one rotation of the star. To test this hypothesis in a simple way we assume a homogeneous element distribution on the stellar surface and an equatorially symmetric rotator model including both dipole 
(Gauss)

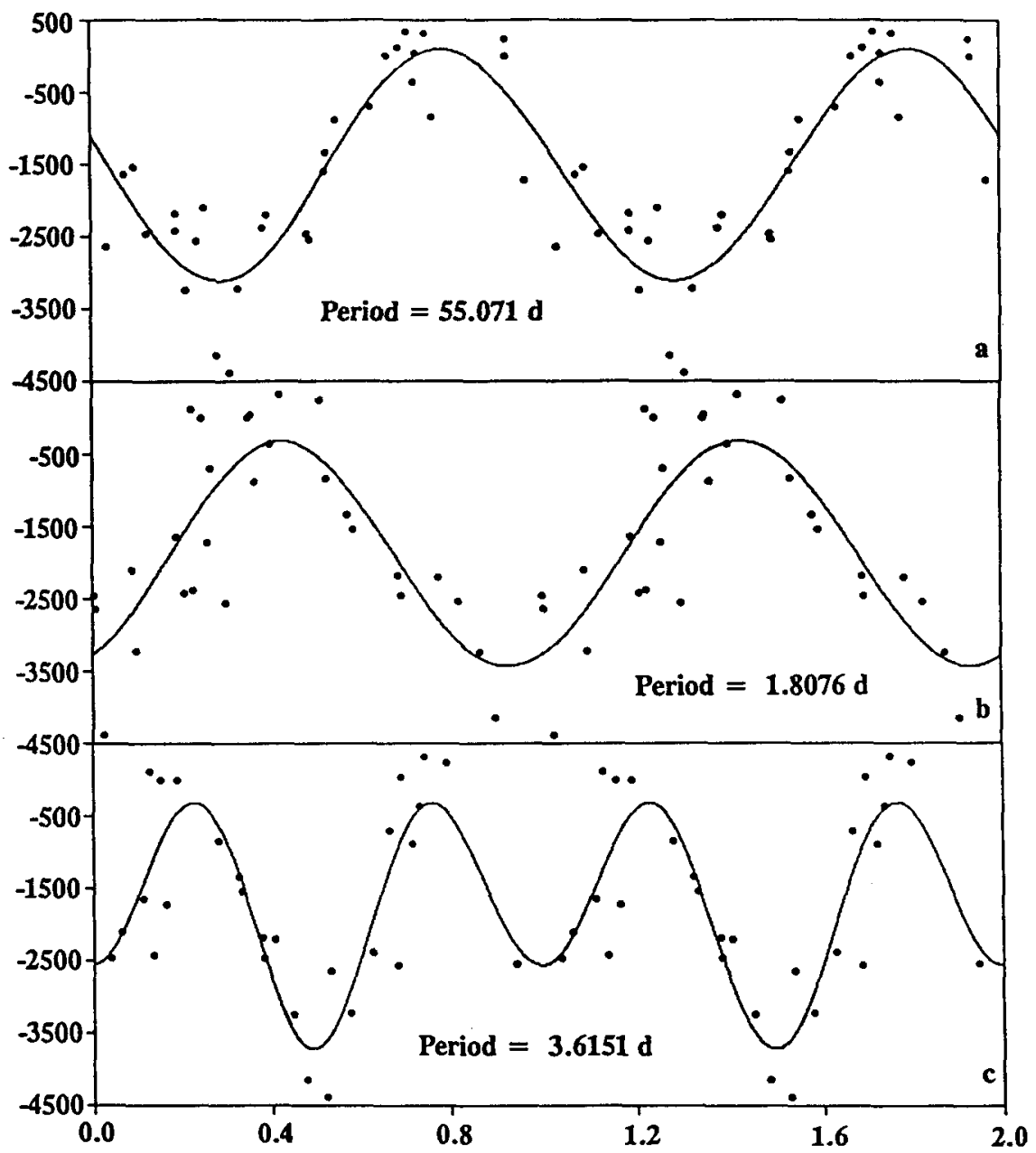

Fig. 2. Effective magnetic field strength versus phase. a) and b) fitting to a sinusoidal variation, c) fitting to equation (1).

$\left(u_{10}\right)$ and quadrupole $\left(u_{20}\right)$ moments (Oetken 1977). Then the observed variation of the effective magnetic field can be described by

$$
\begin{aligned}
& B_{\text {eff }}=C_{1}+C_{2} \cos (2 \pi / P \cdot t)+c_{3} \cos (4 \pi / P \cdot t), \\
& c_{1}=\left(0.08130-0.12195 \mathrm{sin}^{2} i\right) u_{20} / c, \\
& c_{2}=-0.52020 \sin i \cdot u_{10} / c, \\
& c_{3}=-0.12195 \sin ^{2} \cdot u_{20} / c
\end{aligned}
$$


the constants follow from the values for the limb darkening coefficients of $\mu=0.52, x=0.19$ used by oetken. Fig. 2c shows the results of the application of a special period finding technique based on that model. The optimum period is $P=3.6151 \mathrm{~d}$ in good agreement with the period found for the residuals of the radial velocity and the half period of the adopted double wave of $1.8076 \mathrm{~d}$.

From the constants $c_{i}$ the inclination of the rotational axis and the fraction of the dipole and quadrupole moments can be derived: $i=17^{\circ}, u_{10} / u_{20}=0.03$. The values $P_{\text {rot }}=3.6 \mathrm{~d}$, $i=17^{\circ}$ and vsini $=20 \mathrm{~km} / \mathrm{s}$ give a radius of $\alpha$ Dra of $8.9 R_{\rho}$ which is above the typical value of 5-6 $R_{\mathcal{O}}$ for a AO III star. But calling to mind the uncertainties arising from the simple model which allows only an estimation of the inclination as well as from the determination of vini this should be not a principal contradiction to the assumption, that the equatorially symmetric rotator model dominated by the quadrupole field is valid for the giant star $\alpha$ Dra.

\section{REFERENCES}

Adelman, S. J., Bolcal, C., Kocer, D., and Inelmen, E. 1987, Pub. A.S.P., 99, 130 .

Adelman, S. J. 1989, Pub1. A.S.P., 101, 681

Deeming, T. J. 1977, Astrophys. Space Sci., 46, 13

Elst, E. W., Nelles, B. 1983, Astron. Astrophys., Suppl. Ser. 53, 215

Hoffleit, D. 1982, The Bright star Catalogue (4th ed.; New Haven, CT: Yale University Observatory).

Oetken, L. 1977, Astron. Nachr., 298, 197

Slettebak, A., Collins, G. W., Boyce, P. B., White, N. M., and Parkinson, T. D. 1975, Ap. J. Supp1., 29, 137 